\title{
El caso quota hopping en el contexto de la política pesquera común*
}

\author{
Marcos Domínguez Torreiro ${ }^{1}$, Ana B. Freijeiro Álvarez y Manuel Varela Lafuente \\ Departamento de Economía Aplicada. Universidad de Vigo
}

RESUMEN: En este trabajo se analizan las estrategias relativas al proceso de internacionalización de la propiedad dentro del sector pesquero comunitario (en particular la conocida como "quota hopping", aplicada sobre todo por el sector pesquero español). En el desarrollo del proceso ha surgido un conflicto derivado de la defensa del carácter nacional de las cuotas asignadas en la Unión Europea siguiendo el Principio de Estabilidad Relativa. La implantación consiguiente de medidas proteccionistas tales como la exigencia de "vínculos económicos" ha alterado el contexto en que los agentes involucrados en el proceso de internacionalización tomaban sus decisiones. En nuestro artículo analizamos las diferentes estrategias y valoramos los resultados.

PALABRAS CLAVE: quota hopping, política pesquera común, regulación pesquera. CÓDIGOS JEL: Q22, Q18

\section{Quota hopping under the Common Fishing Policy}

SUMMARY: In this article the process of property internationalisation within the fishing sector of the European Union is analysed (in particular, the strategy kwon as quota hopping, as implemented by the Spanish fishing sector). During its development a conflit has arisen in relation with the defense of the national character of the quotas allocated by the Principle of Relative Stability. The implementation of restrictive measures, e.g. the requirement of "economic links" has changed the context where agents involved in the process of internationalisation make their decisions. In our article the different strategies are analysed and the outcomes are evaluated.

KEY WORDS: quota hopping, common fisheries policy, fishing regulation

\footnotetext{
* Los resultados de este trabajo son parte del proyecto de la Unión Europea: Project Fair-CT98-4409, "A legal and economic analysis of foreign ownership of fisheries production in the atlantic" (FOFIP). Asimismo queremos expresar nuestro agradecimiento a José Díaz Carro, Juan Tobío y a ARPEGA por su colaboración, y también a los evaluadores anónimos de este trabajo.

${ }_{1}^{1}$ Marcos Domínguez. Departamento de Economía Aplicada. Universidad de Vigo. Universidad de Vigo. Lagoas - Marcosende s/n 36200-Vigo Tel.: 986.81.40.72 martorre@uvigo.es

Recibido en marzo 2001. Aceptado en noviembre 2002.
} 


\section{Introducción}

Los principios de no discriminación por motivos de nacionalidad, libertad de movimiento de trabajadores y capitales, y libertad de establecimiento, incorporados en el Tratado de la Unión Europea, constituyen la base sobre la cual se ha fundamentado la construcción europea. Estos principios se extienden sobre todos los ámbitos del derecho derivado. Su incorporación en la Política Pesquera Común (PPC) ha tenido lugar bajo la forma del reconocimiento de la igualdad de acceso a las aguas comunitarias por parte de la flota de todos los Estados miembros.

En el caso de la PPC, el derecho de la igualdad de acceso se ha visto sometido a derogaciones de carácter transitorio (doce primeras millas de acceso exclusivo para las flotas del Estado ribereño, Principio de Estabilidad Relativa (PER) y establecimiento de "boxes" de acceso limitado en Irlanda y en las Islas Shetland). El objetivo declarado de las derogaciones es la protección de las comunidades costeras dependientes durante la fase de adaptación de la flota comunitaria a los recursos disponibles. Si bien en este artículo nos centraremos en los criterios territoriales incorporados en la Política Común de Conservación y Gestión de los Recursos, criterios de esta índole han sido incorporados también en los demás ámbitos de la PPC (mercados y estructuras).

A los efectos de nuestro análisis, la restricción más importante a tener en cuenta es el PER, marco general en el que se define la política de gestión de los recursos pesqueros. Esta política aplicada desde 1983 se basa en la definición de Totales Autorizados de Capturas (TACs) por especies, áreas y año, buscando la conservación de los recursos a partir de unidades de gestión y basándose en criterios biológicos. Esos TACs son distribuidos a continuación entre países, a los que se otorgan cuotas en función de un criterio "estable" y "proporcional" de reparto: la estabilidad relativa. Aunque la Comisión Europea ha reconocido ya hace tiempo ${ }^{2}$ las insuficiencias del sistema para superar los problemas de sobrepesca y exceso de esfuerzo, el sistema se ha mantenido en lo esencial. 
La confluencia del principio general comunitario de libertad de circulación y las limitaciones de carácter territorial mencionadas, ha propiciado -entre otras que se han seguido- la estrategia empresarial conocida como "quota hopping"3. El punto central de la estrategia es establecerse en un nuevo país, en el que se compran o abanderan los buques para poder acceder a las cuotas de pesca que ese país tiene asignadas en el marco de la estabilidad relativa.

En el fenómeno del quota hopping hay un conflicto económico latente: el existente entre la eficiencia global y los efectos redistributivos a escala espacial derivados de la actividad. Frente a esta búsqueda de la eficiencia de las empresas que practican esa estrategia ante las rigideces del actual sistema de cuotas nacionales, se encuentra la consideración de los efectos redistributivos y la defensa de la actividad pesquera tradicional en las comunidades costeras dependientes.

Empresas españolas ocupan el primer lugar en términos de penetración en los sectores pesqueros de otros Estados miembros, fundamentalmente Reino Unido y Francia. La búsqueda de acceso a cuotas en especies altamente demandadas por el mercado español, así como el exceso de capacidad pesquera puesto de manifiesto tras las negociaciones previas a la Adhesión, han sido los principales motivos. Actualmente, la situación en la que se encuentran los inversores españoles en otros Estados miembros presenta un alto grado de incertidumbre, dadas las medidas tomadas por los Estados implicados en defensa del carácter nacional de las cuotas (vínculo económico) que amenazan la continuidad de los derechos de pesca y la viabilidad de las empresas. Por otra parte, esta circunstancia no incentiva el desarrollo de actitudes más conservacionistas ${ }^{4}$.

\footnotetext{
${ }^{2}$ Comisión Europea (1991).

${ }^{3}$ La propia denominación "quota hopping" no es muy correcta. En primer lugar, define el todo por la parte, porque se refiere a una estrategia entre las posibles y a un aspecto de un problema más amplio. En segundo lugar, el término resulta algo peyorativo y confuso, al definir lo que es una estrategia económica con una expresión ambigua que califica a los agentes como buscadores o salteadores de cuotas.

${ }^{4}$ Circunstancias como la complejidad de los ecosistemas marinos y la definición poco precisa de los derechos de pesca en la medida en que implican mayor grado de incertidumbre obstaculizan la gestión sostenible de las pesquerías. En la literatura económica de pesquerías se tiende a concluir que al introducir fluctuaciones estocásticas el nivel de stock óptimo puede ser diferente al obtenido en un escenario determinista y que la estrategia apropiada sería más conservacionista. Un planteamiento de referencia es el de Reed $(1979,1988)$. Estos resultados son los que tenemos en cuenta al valorar las posibles conductas de los quota hoppers, aunque naturalmente se puede considerar otros escenarios. Actualmente se han matizado los resultados para stocks medioambientales [Jhohnston and Sutinen (1996), Admunsen and Bjorndal (1999) y Murillas (2001)].
} 
El objetivo de este artículo es analizar la evolución del proceso de internacionalización de la propiedad dentro del sector pesquero comunitario, centrado hasta ahora en la estrategia quota hopping, y las repercusiones tanto del mismo como de las medidas proteccionistas habidas con posterioridad. Estas medidas, tales como la exigencia de "vínculos económicos", han surgido a raíz del temor a los supuestos efectos perjudiciales del quota hopping sobre las economías de las comunidades costeras de los Estados receptores del capital. En cambio, los principios generales de libertad de establecimiento, movimiento y no discriminación son esgrimidos por los partidarios del quota hopping para defender su actuación como legítima. En el artículo realizamos una valoración de resultados para el período considerado en el marco de la discusión actual sobre la regulación pesquera.

Para cubrir estos objetivos el presente artículo queda estructurado de la siguiente forma. En un primer apartado se contextualiza el conflicto del quota hopping dentro del marco de la PPC. En los dos apartados siguientes se realiza un estudio aplicado del fenómeno de la internacionalización de la propiedad en el sector pesquero europeo, desde la perspectiva de España como país exportador y de Francia y Reino Unido como principales receptores. En primer lugar, se analiza la evolución histórica del proceso de creación de empresas pesqueras conjuntas por parte de los empresarios españoles, el cual a su vez se ha visto condicionado por el marco legal vigente en los distintos países implicados. En segundo lugar, se analiza el impacto en las economías locales y el grado de integración de las flotas implicadas dentro de los Estados de pabellón de las mismas, así como los vínculos con las comunidades costeras españolas donde mayoritariamente sitúan sus puertos base. Para la realización de estos dos apartados han jugado un papel fundamental las consultas directas, en forma de entrevistas y cuestionarios, mantenidas con miembros del sector pesquero gallego implicados en el proceso. Por último, se presentan las conclusiones más relevantes.

\section{El quota hopping y los principios fundamentales de la PPC}

Los Reglamentos (CEE) 2141/70 y 101/76 desarrollaron el derecho de igualdad de acceso a las aguas comunitarias para todos los Estados miembros, en iguales 
condiciones de acceso y de uso de las zonas de pesca situadas en las aguas bajo su soberanía o jurisdicción para todos los buques pesqueros abanderados en un Estado miembro y registrados en un territorio de la Comunidad ${ }^{5}$.

Como hemos señalado, el derecho de igualdad de acceso se ha visto sometido a derogaciones, en principio transitorias, pero que con el paso del tiempo parecen tener un carácter indefinido. Esto ha sucedido con la reserva del acceso exclusivo para los nacionales de los países costeros a las doce primeras millas, el acceso restringido a los Boxes de Irlanda y de las Islas Shetland y con el PER, medidas todas ellas recogidas en el Reglamento (CEE) 170/83 y posteriormente trasladadas al 3760/92, actualmente en vigor. El mantenimiento de estas derogaciones da lugar a conflictos e incertidumbres. El ejemplo más claro de los conflictos es el derivado del proceso de internacionalización de la propiedad dentro del sector pesquero comunitario (quota hopping).

El quota hopping se ha incorporado a las agendas de los representantes políticos de los países receptores a través de la presión de los miembros de las comunidades costeras implicadas, cuyas economías tienen un carácter altamente dependiente de la pesca. Estas comunidades se encuentran constituidas por individuos fuertemente vinculados entre sí y el nexo de unión es la actividad pesquera que comparten en la misma región. De estos dos criterios, actividad y territorio, es el último el que prima, puesto que la demanda entre los pescadores europeos para el desarrollo de comunidades transnacionales es mínima (Lequesne, 1998). Actuando en base a los principios propuestos por la perspectiva del "embeddedness", los individuos persiguiendo el interés de la comunidad de la que se sienten parte se han agrupado en "lobbies" que presionan a sus representantes políticos para obtener concesiones que defiendan sus intereses territoriales frente a los de los quota hoppers. Esto es lo que ha sucedido tanto en el Reino Unido como en Francia (Lequesne, 2000). Estamos pues ante un proceso "bottom-up" dentro del diseño de las políticas de gestión de recursos

\footnotetext{
${ }^{5}$ Para un mayor detalle acerca del proceso de construcción europea en el apartado pesquero se pueden consultar Holden (1994) o Karagiannakos (1995).

${ }^{6}$ La cual defiende la actuación de los individuos condicionada por las reglas sociales de carácter informal que los ligan al grupo del que forman parte, llevándolos a comprometerse en la persecución del bien común del mismo (véase Granovetter, 1992, Etzioni, 1988, y Jentoft et al., 1998).
} 
pesqueros $^{7}$, donde la participación activa de los usuarios del recurso colaborando con los organismos gubernamentales en el diseño de las políticas tiene lugar de un modo similar al conocido en la teoría como "comanagement".

El resultado de estas presiones ha sido la aparición en los países receptores de regulaciones que establecen la exigencia de vínculos económicos entre los buques que capturan especies sujetas a cuota y esos Estados de pabellón. La exigencia del vínculo económico es defendida por sus partidarios para respetar el carácter nacional de las cuotas asignadas por la aplicación del PER. Las empresas afectadas por la imposición del vínculo, argumentan que éste es contrario a los principios fundamentales de la Unión Europea, y no hace sino perpetuar las rigideces del actual sistema de cuotas nacionales. En la sección 4 valoraremos beneficios y perjuicios derivados de la aplicación del vínculo.

Por otra parte, detrás del conflicto entre la defensa de los intereses de comunidades locales dependientes de la pesca y la actividad de los quota hoppers se encuentra un enfrentamiento de fondo entre los criterios de distribución territorial y libre mercado. Cabe preguntarse aquí si a través del énfasis en el mantenimiento de la actividad tradicional se están defendiendo intereses perfectamente legítimos por medio de mecanismos proteccionistas, en perjuicio de la eficiencia global del sector pesquero comunitario.

En relación con lo expuesto anteriormente, la búsqueda individual de beneficios motivó el registro y la compra de buques en los Estados receptores por parte de los empresarios españoles. Los propietarios del Estado receptor venden sus buques siempre que con esta venta obtengan un beneficio mayor que el que obtendrían si continuaran desarrollando la actividad pesquera por sus propios medios. Tal y como sucede en este caso, si los beneficios repercutidos de la explotación a cargo de

\footnotetext{
${ }^{7}$ Son los regulados los que proponen la agenda política a los reguladores, al contrario de lo que ocurre habitualmente.

${ }^{8}$ El "comanagement" plantea la conveniencia del reparto de responsabilidades en el diseño y la gestión de los recursos pesqueros entre el Gobierno y los grupos de usuarios. Destacamos, entre otras, las aportaciones teóricas en esta cuestión realizadas por Jentoft (1989), Jentoft et al., (1998), Yamamoto (1995), Iglesias (2000), Gallastegui y Chamorro (1997) y Varela et al. (2000).
} 
extranjeros $^{9}$ superan a los beneficios de una explotación en manos de nacionales, es racional dejar hacer y destinar los recursos nacionales a otros ámbitos económicos (Munro,1985; Cunningham, 2000). En conjunto, la eficiencia global del sector pesquero comunitario ha mejorado, ya que son las unidades más eficientes y rentables las que finalmente explotan el recurso. En el contexto de las rigideces del sistema actual, el quota hopping aparece como sintomático de un deseo de comercio internacional de cuotas (Hatcher et al., 2000).

El Considerando 13 del Reglamento (CEE) 3760/92 recoge la necesidad de "salvaguardar las particulares necesidades de las regiones donde las poblaciones locales sean especialmente dependientes de la pesca”. La interpretación que se ha hecho de esta obligación ha implicado la utilización de criterios tales como el vínculo económico para promover la reserva del carácter nacional de las cuotas frente a la penetración de capitales extranjeros. En el párrafo anterior se ha comentado como esta internacionalización no sólo es beneficiosa para la eficiencia global de la actividad pesquera en el entorno comunitario, sino que además la compraventa de cuota no implica un perjuicio para el bienestar de las comunidades pesqueras dependientes. La cuestión que se plantea bajo esta nueva perspectiva es si las políticas redistributivas pueden seguir persiguiendo la continuidad de la pesca y actividades relacionadas en las comunidades costeras receptoras, o si se deben establecer nuevas políticas a nivel estatal que propongan alternativas locales a la pesca garantizando una renta justa a dichas comunidades $^{10}$. Con ello se lograría poner fin a las trabas que impiden el desarrollo de un mercado libre de derechos pesqueros, permitiendo el desarrollo pleno de los principios rectores de la Unión Europea y la consiguiente creación de un Mercado Único en el sector pesquero, sin que por ello se ocasione un perjuicio ni se menosprecie la protección y desarrollo socioeconómico de las regiones más desfavorecidas.

En las dos secciones siguientes se presenta el análisis de los antecedentes históricos y de los efectos derivados de la aplicación del vínculo económico en las economías receptoras, en este caso Reino Unido y Francia.

\footnotetext{
${ }^{9}$ Los beneficios repercutidos englobarían tanto el importe de la venta de los derechos como el valor adicional de los gastos y suministros que la flota de quota hoppers realice en estas comunidades.

${ }^{10}$ En el propio Libro Verde sobre el futuro de la Política Pesquera Común (Comisión Europea, 2001) se aboga por la reducción de la dependencia por medio del fortalecimiento y diversificación del tejido económico en las zonas dependientes de la pesca.
} 


\section{Evolución histórica}

En esta sección se sintetiza la evolución histórica del proceso de creación de empresas conjuntas pesqueras por parte de los empresarios españoles. Se analizan las características de este proceso de implantación dentro de los sectores pesqueros británico y francés. Cada caso presenta características diferenciadas debido a las distintas regulaciones relativas a la cuestión que han estado en vigor en cada uno de estos países. Entre ellas destacan la aparición de regulaciones acerca de las condiciones de obtención de licencias (vínculo económico), así como aquellas referidas a las condiciones para el registro de buques (Merchant Shipping Act).

A partir de la década de los 60 y fomentado desde la Administración se consolidó en España un sector pesquero orientado a abastecer el mercado de abundante proteína animal a bajo coste. La existencia de libre acceso a los recursos pesqueros marinos aceleró este proceso. Paralelamente al desarrollo de una poderosa flota extractiva, el mercado interno creció hasta convertirse en uno de los principales a escala mundial.

Para la flota española, en 1964 empezaron a surgir las primeras señales de cambio referidas a las posibilidades de acceso al recurso. Ese año se firma el Convenio de Londres ${ }^{11}$ en el que se establece la jurisdicción exclusiva en materia de pesca a los países ribereños hasta las doce millas desde sus costas. Esto no impide el reconocimiento de derechos históricos en la franja comprendida entre las seis y doce millas. Pero era el anticipo del cambio que en la década de los setenta supondría la aparición de las Zonas Económicas Exclusivas (ZEE), poniendo en evidencia a los países que como en el caso de España apostaron fuertemente por flotas de larga distancia.

Con la generalización en 1977 de las ZEE de 200 millas, los Estados ribereños nacionalizan la mayor parte de los recursos pesqueros existentes en el planeta y expulsan de sus aguas a las flotas de larga distancia. La flota española ve así impedida 
la entrada en sus caladeros tradicionales, viéndose obligada a desarrollar una serie de estrategias que permitan mantener la actividad de los buques en los mismos. Propietarios de empresas de capital familiar se ven atrapados por una "inercia institucional" (Jentoft et al., 1998) que les lleva a aceptar el riesgo de invertir y convertirse en quota hoppers. Estas estrategias, apoyadas a nivel de las administraciones públicas, consistieron en buscar nuevas vías de acceso a los recursos:

- Adquisición mediante tratados de colaboración de licencias necesarias para la explotación del recurso del país ribereño.

- Creación de Empresas Pesqueras Conjuntas (EPCs).

Según la legislación vigente en España previa a la Adhesión (Decreto 2517/76) las empresas españolas que participasen al menos en un $40 \%$ de aquellas EPCs, se les concedieron créditos a la exportación de buques, cobertura de riesgos no comerciales y contingentes para la importación de capturas a "tipo cero". Este último beneficio constituyó el principal motivo de éxito y el principal incentivo para la puesta en marcha de empresas conjuntas. Permitió ubicar buques en un caladero de especies de alta demanda y cotización en España e importarlas con el mismo tratamiento que las capturas procedentes de los barcos españoles.

En 1986 España cuenta con 80 empresas conjuntas en todo el mundo, formadas por 205 buques, entre los cuales se encuentran 49 de bandera británica y 9 de bandera irlandesa (Anexo XII del Tratado de Adhesión) ${ }^{12}$. El país comunitario con el que mayoritariamente se establecieron relaciones bajo la fórmula de empresas conjuntas fue Reino Unido. La conjunción de dos factores explica esta situación: la laxitud inicial de la legislación británica al respecto de la incorporación al registro y libre concesión de licencias, y la existencia de un caladero muy rico en especies tradicionalmente demandadas en el mercado español. Se da además la coincidencia de que esas especies (merluza, rape y gallo) tenían entonces menor interés para los pescadores británicos (más interesados en la captura de bacalao, platija y lenguado). Francia introdujo en el momento previo a la Adhesión de España el rape y el gallo como especies sensibles

\footnotetext{
${ }^{11}$ Este convenio es firmado por trece países, entre ellos Francia, Inglaterra e Irlanda.

12 Según el Informe I/1998 del CES, en el periodo 1977-1985 se constituyeron 122 EPCs con 231 buques aportados y un total de 120.418 TRB (el $20 \%$ de la flota pesquera industrial española). En el Anexo XII
} 
(TACs 1984). Reino Unido cedió la mayor parte de las cuotas para estos recursos situados en sus aguas próximas, centrándose en defender las cuotas de las especies más atractivas para su mercado interno ${ }^{13}$. En este punto debemos tener presente que Gran Bretaña e Irlanda obtuvieron cuotas de merluza, rape y gallo en función de la actividad de las antiguas empresas conjuntas hispano-comunitarias.

Con la Adhesión el sistema de incentivos establecido por el Gobierno español para la creación de empresas conjuntas es sustituido por un sistema comunitario homogéneo para todos los Estados miembros, abriéndose un periodo transitorio de siete años para la adaptación de la normativa española a la comunitaria. Durante el mismo se fueron reduciendo progresivamente los aranceles para la importación de productos comunitarios pesqueros (incorporación al Mercado Único).

Dentro del nuevo marco legislativo, continuaron las inversiones de capital a nivel intracomunitario. Dadas las restrictivas condiciones de acceso a zonas y recursos fijadas en el Tratado de Adhesión (lista básica “de los 300” y exclusión del Irish Box) y la fuerte demanda del mercado interno español, continuaban los incentivos para establecerse en otros Estados miembros y pescar con cargo a sus cuotas nacionales ${ }^{14}$. Para el caso de las inversiones españolas en el Reino Unido, tras la Adhesión se produce un cambio de estrategia. Se pasa de la exportación de buques españoles a la adquisición de buques británicos ya registrados. El motivo de este cambio no es tanto la supresión del esquema español de incentivos tras la incorporación a la Comunidad como la creación en 1984 de un sistema restrictivo de licencias en el Reino Unido. Éste inicialmente afectó al rape, para después extenderse a otras especies de interés para los pescadores españoles (gallo, merluza, cigala). Con la extensión del sistema de licencias restrictivas se logró el objetivo de limitar el número de participantes en las distintas pesquerías.

En 1986 se propuso la retirada de la licencia a aquellos buques que no cumplieran tres condiciones relativas a la justificación de un "vínculo económico". Los

\footnotetext{
faltan empresas conjuntas que habían exportado buques al registro británico, pero que no disfrutaban de las ventajas arancelarias, así como buques británicos ya registrados adquiridos por españoles.

${ }^{13}$ El porcentaje de la cuota total comunitaria de rape y gallo asignada al Reino Unido fue respectivamente del 12'3 y 22'72 \%; para Francia fue del 70'76 y 54'54\%.

${ }^{14}$ En los años inmediatamente posteriores a la Adhesión, el número de buques de capital español en el registro británico practicamente duplicó las cifras previas, llegando al centenar de buques.
} 
armadores españoles, argumentando que estas condiciones atentaban contra los principios básicos del Tratado de Roma, acudieron al Tribunal Europeo de Justicia (TEJ) solicitando la supresión de las mismas ${ }^{15}$.

Tras el fallo del TEJ en 1989 y el reconocimiento del derecho de los Estados miembros a exigir un vínculo económico, las autoridades británicas negociaron con los armadores implicados un nuevo vínculo que permitía optar entre dos alternativas: realizar el 50\% de los desembarcos en el Reino Unido o cuatro entradas cada seis meses en puertos británicos. Esta segunda opción sería la elegida por los armadores españoles.

En ese mismo año entra en vigor la Merchant Shipping Act (MSA) que había sido aprobada en 1988. La MSA impuso la obligación de registrarse nuevamente a todos los buques británicos. No fueron admitidos en el nuevo registro todos aquellos cuyas empresas propietarias no cumpliesen los requisitos exigidos ${ }^{16}$. La entrada en vigor de dicha ley afectó a 150 buques que hasta ese momento se encontraban inscritos en el registro británico de buques pesqueros. De esos 150 buques 97 eran españoles.

Tras las denuncias de armadores y gobierno español ${ }^{17}$, el TEJ deja sin efecto los requisitos de nacionalidad exigidos por el gobierno británico ${ }^{18}$. Pero no anuló los de residencia y domicilio, lo que permitió el uso restrictivo de dichos requisitos y mantener en vigor la mayor parte de los artículos de la MSA. Esta intervención del TEJ y la venta de buques hispano-británicos a propietarios que cumplieran esos requisitos permitió la inscripción en el nuevo registro de 32 buques pesqueros de los 150 expulsados.

\footnotetext{
${ }^{15}$ Casos Jaderow (C-216/87) y Agegate (C-3/87).

${ }^{16}$ Estos requisitos consistían en que las operaciones fuesen dirigidas y controladas desde dentro de UK, así como que al menos el $75 \%$ de sus accionistas y directivos tuviesen nacionalidad, residencia y domicilio británico.

${ }^{17}$ Caso Factortame (C-213/89).
} 


\section{CUADRO 1}

Evolución de los buques registrados en el Reino Unido tras la aplicación de la MSA

\begin{tabular}{|c|c|}
\hline Buques registrados a 31-3-89. & $\begin{array}{l}150 \text { buques: } \\
95 \text { buques Factortame* } \\
55 \text { buques no Factortame } \\
\text { (al menos } 6 \text { españoles) }\end{array}$ \\
\hline $\begin{array}{l}\text { Buques re-registrados entre } 1-4-89 \text { a } 3-7-90 \\
\text { (vendiéndolos a otros propietarios o como resultado } \\
\text { de la intermediación del Tribunal Europeo respecto al } \\
\text { requisito de la nacionalidad) }\end{array}$ & $\begin{array}{l}32 \text { buques: } \\
20 \text { buques Factortame } \\
12 \text { buques no Factortame }\end{array}$ \\
\hline $\begin{array}{l}\text { Buques Factortame registrados tras la intermediación } \\
\text { de la Casa de los Lores en Julio de } 1990 .\end{array}$ & $\begin{array}{l}53 \text { buques: } \\
-\quad 48 \text { de los } 95 \text { buques Factortame registrados } \\
\quad \text { antes del } 31-3-89 . \\
-\quad 5 \text { buques no Factortame. }\end{array}$ \\
\hline Buques sobrantes no registrados & 65 buques \\
\hline $\begin{array}{l}\text { Buques Factortame sobrantes no registrados (de los } \\
\text { cuáles } 13 \text { son beneficiarios de la intermediación de } \\
\text { la Casa de los Lores). }\end{array}$ & 25 buques \\
\hline $\begin{array}{l}\text { Buques no Factortame sobrantes no registrados. } \\
\text { (Muchos de ellos desaparecen por hundimiento, } \\
\text { desguace, cambio de bandera o se dedican a } \\
\text { actividades no relacionadas con la pesca) }\end{array}$ & 40 buques \\
\hline
\end{tabular}

*Buques Factortame: buques que denunciaron ante el TEJ su situación en el caso Factortame. Debe su nombre al primero de la lista de denunciantes.

Fuente: Elaboración propia a partir de Karagiannakos (1995).

Finalmente, en 1991 el TEJ dictaminó que la MSA violaba el Tratado de Roma y que la retroactividad introducida al obligar a los buques que ya faenaban en sus aguas a inscribirse en un nuevo registro, era inaplicable. Cualquier requisito de nacionalidad, residencia o domicilio es considerado contrario a la libertad de establecimiento garantizada por el Tratado de Roma ${ }^{19}$. Se establece así una jerarquía de principios en la que la no discriminación estaría siempre por encima de la defensa de las cuotas nacionales bajo el supuesto de estabilidad relativa. El Gobierno británico retiró entonces la ley. A partir de este momento, 53 buques se inscriben en el nuevo registro, de los cuáles 48 habían estado implicados en el caso Factortame.

\footnotetext{
${ }^{18}$ Orden del 20 de octubre de 1989 del Presidente del TEJ.
} 
En resumen, de los 150 buques afectados por la entrada en vigor de la MSA en 1989 sólo 85 buques logran inscribirse en el nuevo registro en 1990. En concreto y para el caso español, de los 97 buques inicialmente inscritos vuelven a registrarse 68 barcos. Por lo tanto, la flota hispano-británica se ve reducida en más de un $25 \%$ tras la aplicación de la MSA.

A comienzos de la década de los 90 se consolida la separación entre "sector" y "no sector"20. Los buques españoles afectados por la MSA, una vez readmitidos en el registro británico, pasan a formar parte del no sector. Se establece un sistema de reparto de las cuotas nacionales entre no sector y sector, y dentro del sector entre las diferentes Organizaciones de Productores (OPs) que lo conforman ${ }^{21}$. Debemos tener en cuenta que en ese momento muchos de los buques afectados por la MSA todavía no habían sido readmitidos en el registro y no podían pescar. Buques británicos dirigieron su actividad en esos años hacia especies de especial interés en el mercado español y que hasta entonces eran capturadas mayoritariamente por los pescadores españoles. De esta forma los buques británicos adquirieron unos "derechos históricos" durante ese intervalo en detrimento de los buques que se encontraban paralizados forzosamente. Aquellos buques se hicieron con una mayor proporción de cuotas, reduciéndose la parte correspondiente a los buques que habían vuelto tras la expulsión.

Desde entonces, la mayor parte de los buques hispano-británicos están integrados dentro del sector. La integración en el seno de una OP implica una serie de ventajas: posibilidad de reparto individualizado de cuotas y de su intercambio temporal dentro de la misma OP o entre distintas OPs. La presencia de estas ventajas, así como la progresiva reducción de las cuotas asignadas al no sector, llevaron en 1992 a que buques de capital español se asociaran y crearan su propia OP (Wales and West Coast Fish Producers' Organisation), contando en 1996 con 47 miembros (Hatcher, 1997) ${ }^{22}$. Años más tarde muchos de los restantes buques se integraron en una OP ya en funcionamiento (Fleetwood Fish Producers' Organisation).

\footnotetext{
${ }^{19}$ La única condición que no se consideró contraria a la Ley comunitaria fue la de que las operaciones fueran dirigidas y controladas desde dentro del Estado miembro.

${ }^{20}$ Se denomina "sector" al conjunto de buques integrados en las distintas Organizaciones de Productores británicas, y "no sector" a todos aquellos buques que se encuentran al margen de las mismas.

${ }^{21}$ Este reparto se calcula en base al "track record" de los desembarcos realizados por cada buque durante los tres últimos años.

${ }^{22}$ Según Billón Currás (1998), en 1997 hay 70 buques que operan desde Milford Haven, en Gales.
} 
En 1997 Gran Bretaña cambió las exigencias para cumplir con el vínculo económico. Unas nuevas condiciones legales se han incorporado a las licencias de pesca británicas en 1999, condiciones que ponen en peligro la viabilidad de muchas empresas. La normativa en vigor desde el 1 de enero de 2000 propone ahora cuatro opciones para demostrar la existencia de un vínculo económico relevante entre las empresas pesqueras y el Reino Unido:

A) Descargar en el Reino Unido al menos el 50\% de las capturas sometidas a cuotas.

B) Emplear una tripulación en la que al menos el 50\% de sus miembros sean residentes en una zona costera del Reino Unido.

C) Incurrir en gastos en el Reino Unido iguales, al menos, al 50\% del valor neto de las descargas de especies sujetas a cuota o al $50 \%$ de los gastos de explotación.

D) Demostrar por otros medios el vínculo, incluidas combinaciones de las opciones anteriores. La aceptación de estas combinaciones, o nuevas fórmulas queda a la discrecionalidad de las autoridades británicas. La posibilidad de utilizar dos o tres condiciones conjuntamente, exige el cumplimiento del $75 \%$ de los requisitos exigidos.

Además de dicho vínculo económico, se exige que todos los barcos que descarguen una cantidad inferior al 50\% de sus capturas de especies sujetas a cuota, realicen al menos una entrada en un puerto del Reino Unido cada seis meses. Dicha entrada exige una permanencia mínima de ocho horas para facilitar inspecciones de capturas, artes de pesca y documentos. Junto con esta "visita obligatoria" continúa en vigor el requisito de nacionalidad en el que se exige que al menos el $75 \%$ de la tripulación sea comunitaria.

Con relación a la situación actual es interesante analizar también la evolución histórica de la exportación de capital pesquero español a Francia. Desde la extensión de las ZEEs en 1977 no se tiene constancia de la exportación de ninguna unidad pesquera a Francia para la constitución de una empresa pesquera conjunta. La estrategia del capital español para acceder a las cuotas francesas fue la de adquisición de 
unidades pesqueras ya registradas y con licencia. Esto es algo distinto a lo que estaba ocurriendo en el Reino Unido, donde la incorporación en el registro de buques pertenecientes a empresas conjuntas que anteriormente habían pertenecido al registro español fue una estrategia habitual desde la extensión de las ZEEs comunitarias a 200 millas. La explicación de esta diferencia puede estar en el énfasis que en la regulación francesa se hace en el factor de la presencia histórica en la pesquería para la concesión de licencias (antèrioritès de pêche). Si a esto se suma la holgura de las cuotas asignadas a Francia para cubrir las capturas realizadas por su flota nacional de especies que interesan a la flota de capital español (principalmente merluza y rape), se justifica que el interés de las autoridades francesas por la constatación de un vínculo económico tenga fechas tan recientes.

El vínculo económico en el caso francés se implanta más tarde con la aprobación de la Ley 97/1051 de Noviembre de 1997, en la cuál se establecen las vías para demostrar el vínculo económico entre el barco y el territorio francés y la existencia de una "sede social estable". Fue 1999 el primer año en el que se exigió el cumplimiento de estas condiciones. La consecuencia inmediata fue la retirada de cuotas por incumplimiento del vínculo a 15 pesqueros franco-españoles a finales de ese mismo año. Las alternativas existentes para el cumplimiento del vínculo son las siguientes:

A) Descarga del $50 \%$ de las capturas y vender una parte sustancial de las mismas en puerto francés ${ }^{23}$.

B) Que el 50\% de la tripulación residente en una zona pesquera francesa.

C) Que la mitad de las faenas de pesca partan de puerto francés.

D) Otros elementos de prueba de peso económico y estructural equivalente que demuestre la existencia de un vínculo real.

\section{Efectos derivados de la aplicación del vínculo económico}

Una vez comentada la evolución histórica de este proceso, en esta sección se analizan los efectos económicos derivados de la aplicación del vínculo en el

\footnotetext{
${ }^{23}$ Lo que supone un paso adelante respecto a la normativa británica con la introducción de la venta obligatoria
} 
Reino Unido y Francia. Para este análisis han jugado un papel fundamental los datos y opiniones obtenidos por medio de entrevistas y cuestionarios realizados a empresarios españoles propietarios de buques registrados en estos dos países, directamente afectados por la aplicación del vínculo económico ${ }^{24}$

En el Reino Unido, el vínculo económico ha cumplido su objetivo de redistribuir a las comunidades costeras británicas parte de las rentas procedentes de la explotación por los quota hoppers de recursos pesqueros sujetos a cuota comunitaria y asignados a ese Estado. Esta redistribución se ha logrado a costa de restringir los criterios de eficiencia en la actividad extractiva de la flota. Considerando pues en su totalidad los efectos derivados de la implantación del vínculo, se puede poner en duda su conveniencia.

La redistribución de rentas hacia las comunidades costeras británicas proviene principalmente del cumplimiento de la condición de desembarcar al menos el $50 \%$ de las capturas de especies sometidas a cuota en el Reino Unido, pagando las correspondientes tasas. Esta ha sido la opción mayoritariamente elegida para el cumplimiento del requisito del vínculo por los quota-hoppers españoles.

El beneficio derivado de los desembarcos realizados por la flota de capital español en puertos británicos no se ha visto reforzado por una actividad de venta de los mismos en lonjas británicas. Se advierte una dualidad entre puertos de desembarco y lugar de comercialización. Si bien los desembarcos en puertos británicos pueden llegar a suponer un porcentaje significativo de las capturas (en torno al 50\%

\footnotetext{
24 En los cuestionarios se han incluido preguntas referentes a las características de los buques, datos técnicos de la actividad pesquera, motivaciones iniciales para desarrollar la actividad y efectos de las medidas actualmente en vigor sobre la viabilidad futura de la misma. Estos cuestionarios fueron realizados sobre una muestra aleatoria simple del $10 \%$ de la flota quota hopping británica y francesa con base en Galicia ( 90 buques gallegos sobre un total de 140 buques con base en España). Por facilidad en la realización de las entrevistas se ha acudido únicamente a armadores gallegos, si bien esto no supone una pérdida de representatividad en la selección de la muestra dada la homogeneidad presente en los buques y en las estrategias de los mismos a lo largo de todo el territorio español. Se trata de buques especializados en las mismas especies demersales, con el mismo número de tripulantes a bordo, que faenan en las mismas áeras, con "mareas" de idéntica duración y que se encuentran concentrados en unos pocos puertos base. Para un nivel de confianza del 95\%, el error muestral en términos de potencia se sitúa en torno al $10 \%$ del valor promedio de la flota. Para el cálculo del error muestral hemos utilizado la siguiente fórmula:

$$
n=\frac{N \cdot k^{2} \cdot s^{2}}{N \cdot e^{2}+s^{2} \cdot k^{2}}
$$

donde $n$ es el tamaño de la muestra, $N$ es el tamaño de la población, $k$ es el parámetro relacionado con el nivel de confianza, $s$ es la desviación típica de la muestra y $e$ es el error muestral.
} 
para aquellos buques que han elegido la primera opción para el cumplimiento del vínculo), el mercado de destino de este pescado se encuentra en España. De igual modo, a pesar de llevar a cabo una parte importante de los desembarcos en puertos británicos, estos buques realizan la mayor parte de los gastos de equipamiento ${ }^{25}$ en sus puertos base españoles (alrededor del 60\%), rechazando la opción de cumplir con el vínculo incurriendo en estos gastos en territorio británico.

Encontramos una situación similar a la anterior cuando se analizan los resultados del vínculo económico en términos de generación de empleo en las zonas británicas dependientes de la pesca. Una de las alternativas propuestas por el gobierno británico para cumplir con el vínculo consiste en que al menos el 50\% de la tripulación de estos buques debe residir en un área costera británica. Sin embargo, podemos comprobar como los armadores de los buques hispano-británicos tampoco eligen esta opción. La mayor parte de la tripulación de estos buques tiene nacionalidad española (llegando al $100 \%$ en los buques de determinadas empresas) y en menor medida, nacionalidad portuguesa.

Llegados a este punto, el siguiente paso de nuestro análisis será valorar si los beneficios derivados de la aplicación del vínculo económico suponen una cuantía relevante para el bienestar de las comunidades locales. En cuanto a los ingresos derivados del desembarco, venta en subasta y equipamiento de los buques, aún suponiendo que la flota de quota hoppers los realizara en su totalidad en el Reino Unido, el montante total de los mismos, según estimaciones recogidas en Hatcher et al. (2000), no rebasaría una cifra estimada de 1'5 millones de libras, cifra relativamente pequeña en comparación con la riqueza generada dentro de la economía de las comunidades costeras británicas.

Por otro lado, un endurecimiento de las condiciones relativas al lugar de desembarco causaría un perjuicio sobre la propia actividad de los buques de bandera británica y capital estrictamente británico. Según estimaciones realizadas en el Reino Unido (Hatcher et al., 2000) cerca de dos tercios del total de desembarcos realizados en

\footnotetext{
${ }^{25}$ Dentro de los gastos de equipamiento se englobarían varios subapartados: combustible, hielo, víveres, material de pesca y reparaciones menores. El gasto realizado en territorio británico para estos distintos subapartados oscila entre prácticamente el $0 \%$ de las reparaciones menores y el $40 \%$ del gasto en combustible.
} 
el extranjero por la flota británica se deben a la actividad de buques de propiedad británica. Aún cuando se les obligase al desembarco de las capturas en puertos británicos, el destino final de estos productos se encuentra en gran parte en el extranjero, donde los precios de venta son mayores. Estos datos ponen de manifiesto el peligro potencial de imponer restricciones de este tipo a la libre toma de decisiones empresariales en base a los criterios de rentabilidad y eficiencia. Los costes adicionales derivados del cumplimiento de estas condiciones (transporte de las mercancías, pérdida de ingresos por venta) empeorarían los resultados de explotación de estas empresas y supondrían una restricción para la viabilidad de las mismas, lo cual repercutiría negativamente sobre el bienestar de las comunidades locales británicas de las que proceden. En cuanto al empleo a bordo, sería difícil encontrar en las zonas costeras británicas pescadores nacionales en número suficiente para cubrir todos los empleos disponibles en la flota de quota hoppers.

\section{CUADRO 2}

Desembarcos realizados por buques británicos (miles de Toneladas)

\begin{tabular}{lccc}
\hline & Desembarcos en UK & $\begin{array}{c}\text { Desembarcos en el } \\
\text { extranjero }\end{array}$ & $\begin{array}{c}\text { \% de desembarcos en el } \\
\text { extranjero sobre el total }\end{array}$ \\
\hline Bacalao & 72.7 & 4.5 & $5.8 \%$ \\
Eglefino & 82.8 & 0.6 & $0.7 \%$ \\
Platija & 11.5 & 12.0 & $51.1 \%$ \\
Lenguado & 2.0 & 0.6 & $23.1 \%$ \\
Rape & 18.5 & 3.2 & $14.8 \%$ \\
Gallo & 5.3 & 1.3 & $20.0 \%$ \\
Merluza & 2.5 & 2.6 & $50.0 \%$ \\
Arenque & 39.5 & 65.1 & $62.2 \%$ \\
Caballa & 54.4 & 125.3 & $69.7 \%$ \\
\hline Total & 428.2 & 362.9 & $45.9 \%$ \\
\hline
\end{tabular}

Fuente: Hatcher et al. (2000).

Hasta ahora hemos visto el papel del vínculo como garante de la repercusión de un cierto bienestar derivado de la explotación de las cuotas nacionales sobre las comunidades pesqueras del Reino Unido. Sin embargo, cuando se presionó desde las comunidades británicas dependientes de la pesca para establecer un vínculo económico, no se reconoció el beneficio repercutido sobre estas comunidades por medio 
de la venta de los buques y derechos a empresas extranjeras. En el precio de venta de los mismos ya va implícita la consecución de una ganancia de bienestar. Los iniciales propietarios de los buques y derechos se desprendieron de ellos libremente porque consideraron que con esta venta obtendrían mayor beneficio que si hubiesen continuado desarrollando la actividad pesquera por sus propios medios. La venta de los activos y el abandono de la actividad de modo voluntario plantea la conveniencia y necesidad del vínculo desde una nueva perspectiva. Las decisiones de abandono voluntario de la actividad son una señal de la necesidad de reorientación de las políticas económicas redistributivas hacia la definición de estrategias económicas que propongan alternativas a la pesca en las comunidades tradicionalmente dependientes. Los compradores, en este caso empresarios españoles, adquirieron estos buques y licencias puesto que consideraban que de la explotación de los mismos obtendrían una mayor rentabilidad que el precio pagado. Estas previsiones de ganancias se realizaron bajo la hipótesis de poder desarrollar su actividad empresarial libremente. El reciente desarrollo de legislaciones que exigen el cumplimiento de un vínculo económico y la incertidumbre acerca de la evolución futura de las condiciones que llevan aparejadas suponen un riesgo para alcanzar las cotas de rentabilidad esperadas de estas inversiones.

Para las empresas españolas el cumplimiento del vínculo ha supuesto restricciones para la eficiencia, rentabilidad y la continuidad de su actividad. Los obstáculos que el vínculo económico implica para los buques dependerán de la condición escogida para cumplir con los requisitos del vínculo. Aquellos empresarios que han elegido la opción del $50 \%$ de los desembarcos, por considerarla como mal menor o la única alternativa viable, señalan que preferirían elegir libremente dónde y en qué porcentajes desembarcar sus capturas, en función del criterio de rentabilidad de su actividad. En el mercado español los precios son superiores a los del mercado británico, por lo que los armadores prefieren transportar el pescado hacia España y soportar este coste de transporte antes que intentar colocar sus capturas en otros mercados menos atractivos. Su estrategia sería entonces la de desembarcar la mayor parte de sus capturas en puertos españoles, ya que es ahí donde tienen su mercado.

En cuanto a la condición de emplear una tripulación compuesta al menos por un $50 \%$ de residentes en una zona costera británica, los armadores españoles de buques hispano-británicos afirman que no existe suficiente mano de obra disponible en 
el Reino Unido para hacer frente a las necesidades de tripulación de esta flota. El mal estado de los buques y su avanzada edad, además de los problemas de productividad y el riesgo que provoca para el desempeño diario de la actividad pesquera, acrecienta los problemas para encontrar tripulación que esté dispuesta a trabajar en estas condiciones, incluso en España ${ }^{26}$.

Los armadores españoles no consideran rentable la adquisición de suministros en puertos británicos, y por ello limitan los gastos de aprovisionamiento a realizar en el Reino Unido a los indispensables en función del plan de actividad en aguas del Gran Sol previsto para el buque. Esto se debe a que todos los suministros necesarios para el funcionamiento de estos buques tienen un precio más elevado en UK en relación a España. Por ello, intentan que la mayor parte de los suministros sean adquiridos en España, concretamente en los puertos base de la flota.

La exigencia de un vínculo económico por parte de los países en cuyos registros se ha instalado esta flota debe ser valorada también en términos de la repercusión que esto tiene sobre las regiones dependientes de la pesca en los países de procedencia del capital. En este sentido es importante resaltar que muchos de los buques de capital español tienen sus puertos base en Galicia. Y para esta comunidad autónoma, el cese o disminución en la actividad de la flota mencionada supondría pérdidas económicas significativas, dada la dimensión de la flota implicada.

En términos de la importancia de la pesca, Galicia ocupa el primer lugar dentro del Estado español. A pesar de haberse ido reduciendo el número total de empleos en el sector pesquero en España y en Galicia, la participación de Galicia en el empleo total de la pesca en España se ha mantenido en torno al 40\%. En cuanto al Valor Añadido Bruto a coste de factores (VABcf), la pesca marítima en Galicia supone un $40 \%$ del total español ${ }^{27}$. Dentro de la propia región, la producción pesquera representa el 4,58 del PIB a precios de mercado, y el número de empleos en el sector el 4,12\% del total de empleos en Galicia.

\footnotetext{
${ }^{26}$ En la actualidad, la flota española ("flota de los 300") cuenta con buques nuevos en muy buenas condiciones. Por ello, los pescadores prefieren trabajar en los buques de la flota española antes que en los buques hispano-británicos. Esto está dificultando todavía más la labor de encontrar una tripulación válida y competente para estos buques.

${ }^{27}$ Fundación BBV (1999).
} 
La flota de Gran Sol registrada en el Reino Unido y con base en Galicia tiene una alta relevancia dentro del total de la flota gallega. En la actualidad se sitúa en torno a 75 buques, lo que representaría un 7’6\% del TRB (Toneladas de Registro Bruto) de la flota gallega, con 258’1 TRB y 14 tripulantes de promedio por buque.

\section{CUADRO 3}

Peso relativo de la flota de quota hoppers con base en Galicia

\begin{tabular}{lccc}
\hline & Número de buques & TRB & $\begin{array}{c}\text { Número de } \\
\text { tripulantes }\end{array}$ \\
\hline Flota Británica & 7.600 & 209.600 & 16.000 \\
Flota Gallega & 8.811 & 254.279 & 28.014 \\
Quota Hoppers* & 75 & 19.361 & 1.050 \\
\hline
\end{tabular}

*Datos estimados.

Fuente: Elaboración propia a partir de datos de la Consellería de Pesca, Marisqueo e Acuicultura y del Annual Report 2000 de la Concerted Action (FAIR PL 97-3541).

Complementando lo visto, el análisis de la situación en Francia apunta a que el vínculo económico ha logrado su objetivo de redistribuir a las comunidades costeras francesas parte de las rentas procedentes de la explotación por los quota hoppers de recursos pesqueros sujetos a cuota comunitaria y asignados a Francia. Y de igual modo que sucede en el Reino Unido, los armadores españoles implicados se manifiestan contrarios a la imposición de vínculos económicos por motivos idénticos. Y los costes de eficiencia derivados de la aplicación del vínculo, se contrapone al argumento anterior.

Dada la similitud entre las condiciones del vínculo exigidas en Francia con las exigidas en el Reino Unido, podemos asumir que el potencial redistributivo de la aplicación de las mismas es, como hemos visto más arriba, limitado. Del mismo modo, los costes en términos de eficiencia y pérdida de rentabilidad para los buques quota hoppers españoles se justifican por los mismos motivos expuestos para el caso británico.

Asimismo, las opciones elegidas para cumplir con las exigencias del vínculo representan la misma significación (diferencial de precios favorable a España 
para la comercialización ${ }^{28}$, difuciltades para enrolar tripulación francesa ${ }^{29}$, restricciones a la libertad de decisión empresarial en general $^{30}$ ).

\section{Conclusiones}

Desde sus orígenes la PPC ha incorporado en sus regulaciones medidas discriminatorias en base a criterios territoriales que se oponen a los principios básicos de libre mercado y no discriminación, rectores del proceso global de construcción europea. Estas medidas, tales como el reparto de cuotas nacionales en base al PER y la exigencia de vínculos económicos, se han incorporado con el objetivo de salvaguardar las particulares necesidades de las regiones especialmente dependientes, tratando de garantizar la continuidad de la actividad pesquera ligada a las comunidades locales.

En el contexto de libertad de mercado, la actividad de los quota hoppers puede implicar una mejora en el nivel de la eficiencia global del sistema. Y por otra parte el efecto redistributivo global resultante de la aplicación de medidas restrictivas tales como el vínculo económico, resulta incierto. Por un lado se ha conseguido una repercusión positiva sobre el bienestar de las comunidades dependientes en los Estados receptores de la flota de quota hoppers, y por otro la exigencia de vínculos económicos ha tenido efectos perjudiciales sobre las comunidades dependientes en los países de origen.

Las políticas redistributivas incorporadas en las medidas destinadas a proteger el bienestar de determinadas regiones por medio del mantenimiento de la actividad pesquera en las mismas deben ser reconsideradas. La liberalización del mercado de derechos de pesca, en vista de lo acontecido con la actuación de los quota hoppers, no tiene por qué considerarse necesariamente una amenaza para estas regiones $^{31}$. En principio debe contribuir a reducir los niveles de incertidumbre, y por

\footnotetext{
${ }^{28}$ Tal sería el caso de los puertos españoles por encontrarse ahí los mercados principales, e incluso de puertos irlandeses por cuestiones de proximidad a los caladeros (tal es el caso del puerto de Castletown).

29 Alguno de los entrevistados incluso opina que de imponerse las mismas condiciones en los buques españoles que el resto de los de la comunidad, los buques hispano-franceses no serían rentables. Sería un argumento para explicar que los armadores españoles no hayan recurrido a la opción de cumplimiento del vínculo relativa a la nacionalidad de los tripulantes.

${ }^{30}$ Por otra parte, cabe destacar la postura de uno de lo entrevistados a favor de la necesidad de defender las economías locales de las zonas francesas altamente dependientes de la pesca. Sin embargo, el mismo entrevistado afirma que el vínculo económico impuesto en la actualidad no se ajusta a la realidad de una empresa económicamente viable.

${ }^{31}$ Desde finales de los 80 se intensificó la discusión sobre los sistemas de regulación en pesca, a la vista de la experiencia de las licencias y cuotas, especialmente. El acento se puso en la mejora de definición de derechos de pesca y ello desde diferentes perspectivas. La consideración dominante es que el desarrollo
} 
tanto debe favorecer el comportamiento conservacionista. Y desde otra perspectiva debe favorecer también a la eficiencia y el ajuste entre esfuerzo (empezando por la dimensión de la flota) y recursos.

Adicionalmente, la protección de los niveles de rentas de sus habitantes no tiene por qué vincularse necesaria o exclusivamente al mantenimiento de la actividad pesquera. Las políticas redistributivas a nivel comunitario no tienen por qué significar el bloqueo a la actividad de los quota hoppers dentro de los países receptores, sino más bien implicar la búsqueda y la elaboración de estrategias de desarrollo local alternativas y coherentes con el avance en el proceso global de construcción europea. $\mathrm{O}$, en otras palabras, aplicar para el sector pesquero, los principios generales de funcionamiento de la economía europea.

\section{Bibliografía}

Amundsen, E. S. And Bjorndal, T. (1999): Optimal Exploitation of a Biomass Confronted with the Threat of Collapse, Land Economics 75: 185-202.

Billón Currás, M. (1997). El Conflicto Pesquero Hispano Británico en el Marco de la Política Común de Pesca. Economía Agraria, 180: 225-262.

Comisión Europea (1991). Informe de la Comisión al Consejo y al Parlamento Europeo sobre la Política Pesquera Común. SEC(91) 2288 final. Bruselas.

Comisión Europea (2001). Libro Verde sobre el futuro de la Política Pesquera Común. $\operatorname{COM}(2001)$ 135. Bruselas.

Cunningham, S. (2000). Fishing agreements: trade and fisheries management. Presentado en el Workshop on International Relations and the Common Fisheries Policy, Bergen, Norway, Octubre.

Etzioni, A. (1988). The moral dimension. Toward a new Economics. The Free Press, New York.

\footnotetext{
de un mercado de derechos contribuiría a mejorar la eficiencia (el ajuste entre esfuerzo y recursos en particular) vía inputs o vía outputs (Neher et al, 1989; Varela Lafuente et al, 1995). Otra perspectiva institucionalista, apunta en la dirección de considerar la implantación de la definición de derechos, no tanto vía mercados sino en la medida en que el papel de las organizaciones de pescadores ( a través de sistemas de autogestión o regimenes de propiedad común) puedan contribuir al ajuste citado a través de reglas diseñadas a nivel de acción colectiva. (Schlager y Ostrom 1992; Suris et al, 1995; Iglesias, 2000).
} 
Gallastegui, M. C., Chamorro, J. M. (1997). Nuevos avances en la gestión de pesquerías. Papeles de Economía Española 71: 196-213.

Granovetter, M. (1992). Economic action and social structure. The problems of embeddedness, en Granovetter, M. y Swedberg, R (eds.).: The Sociology of Economic Life. Westview Press, Boulder, CO: 53-58.

Hatcher, A. (1997). Producers' organizations and devolved fisheries management in the United Kingdom: collective and individual quota systems. Marine Policy 21(6): pp. 519-533.

Hatcher A., Pascoe, S., Robinson, K., Frere, J. (2000). "Quota-hopping” and the foreign ownership of UK fishing vessels. Presentado en el Workshop on International Relations and the Common Fisheries Policy, Bergen, Norway, Octubre.

Holden, M. (1994). The common fisheries policy. Fishing New Books, Oxford.

Iglesias, C. (2000). Análisis económico de la regulación de pesquerías en la Europa Azul: experiencias y alternativas. Tesis Doctoral. Departamento de Economía Aplicada. Universidade de Vigo.

Jentoft, S. (1989). Fisheries comanagement. Delegating government responsibility to fishermen's organizations. Marine Policy 13(2): 137-154.

Jentoft, S., McCay, B.J., Wilson, D.C. (1998). Social Theory and Fisheries Comanagement. Marine Policy 22(4-5): 423-436.

Johnstone, R. J., Sutinen, J. G. (1996): Biomass shift and collapse: Implications for harvest policy in the fishery, Land Economics 72: 500-518.

Karagiannakos, A. (1995). Fisheries management in the European Union. Avebury, Hants (UK).

Lequesne C. (1998). Market Vs. Local Comunities?: Quota Hopping and the EU Fishery Policy. Presentado en la European Consortium for Political ResearchInternational Studies Association Joint Conference, Viena, 16-18 Septiembre.

Lequesne C. (2000). Quota Hopping: The Common Fisheries Policy between States and Market. Journal of Common Market Studies 38(5): 779-93.

Munro, G. (1985). Coastal states, distant water fleets and EFJ: some long run consideations. Marine Policy 9: 2-15.

Murillas Maza, A. (2001): Gestión y valoración de una pesquería con incertidumbre biológica y económica bajo propietario único y propiedad común. Tesis Doctoral. Universidad del País Vasco. 
Neher, P., Arnason, R., Mollet, N. (eds.) (1989): Rights Based Fishing. Kluwer Academic Publishers (Netherlands).

Reed, W. J. (1979): Optimal escapement levels in stochastic and deterministic harvesting models, Journal of Environmental Economics and Management 6: 350-363.

Reed, W. J. (1988): Optimal harvesting of a fishery subject to random catastrophic colapse, IMA Journal of Mathematics Applied in Medicine and Biology 5(4): 215-235.

Schlager, E., Ostrom, E. (1992): Property Rights Regmes and Natural Resources. A Conceptual Analysis, Land Economics, 68(3): 249-262.

Suris, J., Varela, M., Garza, M. D. (1995): Propiedad y soluciones pesqueras, Revista de Economía Aplicada 7: 5-25.

Varela, M.; Surís, J., Garza, M. D., Iglesias, C. (2000). Economía de la pesca. Presente y futuro de la regulación pesquera en Galicia. Fundación Pedro Barrié de la Maza, A Coruña.

Varela, M., Garza, M. D., Iglesias, C. (1996): Gestión de pesquerías basada en derechos de pesca. El caso español en materia de regulación pesquera, Información Comercial Española, 755: 139-156.

VV. AA. (2000). Economic Performance Of Selected European Fleets. Annual Report 2000. Concerted Action (FAIR PL 97-3541). Doc. No. 14.- Final.

Yamamoto, T. (1995). Development of a community-based fishery management system in Japan. Marine Resource Economics, 10: 21-34. 